\title{
MYTHos Cage
}

herausgegeben von Claus-Steffen Mahnkopf

Hofheim: Wolke Verlag, 1999

Rezensionsartikel von Clemens Gresser, Southampton

$-1-$

Mit MYTHos Cage ist eine Sammlung von zehn Beiträgen betitelt, die 1999 im Wolke Verlag erschien. Die Publikation geht auf eine Initiative von Claus-Steffen Mahnkopf zurück, der sich — wie es im Vorwort heißt im Frühjahr 1997

\begin{abstract}
„an einige Autoren im In- und Ausland mit der Bitte [...wandte], auf ihre Weise auf den 'Mythos' Cage zu reagieren. Der vorliegende Band versammelt die Ergebnisse. Deren Diversifität ist willkommen und zeigt einen unaufgeregten, nüchternen Zugang zu einem 'Phänomen', das eher blind-emotionale Gefolgschaft und projektive Gedankenflucht auszulösen vermochte als eine sachbezogene Analyse oder eine unorthodoxe Aneignung mit Kreativität." (S. 7)
\end{abstract}

Solch ein Vorhaben war längst überfällig. Mehr als fünf Jahre nach Cages Tod (1992) sollte eine kritische Auseinandersetzung mit den ihn umgebenden Mythen leichter fallen.

$$
-2-
$$

Leider gelingt es kaum einem der Autoren noch der Autorin in MYTHos Cage, Mythen aufzudecken, vielmehr werden existierende Vorurteile und Einschätzungen bestätigt. Es scheint, als ob die Mehrzahl der Autoren, die Autorin und der Herausgeber ein sehr starres, vorurteilbehaftetes Heiligenbild des Komponisten vor Augen haben und denken, daß dieses mit aller möglichen Wortgewalt und teils großem Seitenumfang zerschlagen werden muß. Eines der Hauptprobleme des Buches ist, daß die meisten Autoren und die Autorin ihre Aussagen nicht genügend argumentativ belegen - beispielsweise mittels Bezug auf Äußerungen Cages oder auf spezifische Kompositionen. In vielen Beiträgen wimmelt es von Unwissenheit, was insbesondere die Kenntnis von kritischwissenschaftlichen Publikationen zu Cage anbelangt. Außerdem haben nur wenige Beiträge eine klare, stringente Struktur - manche Autoren reihen 
Bemerkungen lediglich aneinander und verlieren dabei den Fokus ihres Textes. Aber nicht nur das: Oft scheinen Kompositionen nur aus zweiter Hand bekannt zu sein. Ausgiebig wird verallgemeinert, so daß die Lektüre dieses Buches für wissenschaftliche Zwecke überwiegend unbrauchbar ist. Oft wird von den Kompositionen Cages, der (hagiographen) CageForschung und von der Cage-Philosophie geschrieben. Dazu im folgenden jeweils einige Sätze zur Gegendarstellung: 1. Cages Euvre mit einem Charakteristikum beschreiben zu wollen, muß fehlschlagen, da sein Schaffen zu vielfältig ist: Es besteht aus traditionell notierten Werken (sogar nach 1950), aus graphischen Folien-Kompositionen (beispielsweise Variations IV, 1963), Textnotationen (0'00“, 1962), aus den der Syntax beraubten Adaptionen bereits existierender Kompositionen (Cheap Imitation, 1969), aus Kompositionen, die auf Zufallsoperationen basieren (fast totaldeterminiert wie in Music of Changes, 1952, oder auch flexibel wie 108,1991$)$ sowie aus Werken, die traditionell notiert sind und auf komplexeren, proportionalen Strukturen beruhen (First Construction in Metal, 1939); und selbst diese Aufzählung ist keinesfalls vollständig. Wie kann bei solch einem heterogenen Euvre von den Kompositionen die Rede sein, ohne daß die Applikation der Aussage als Verallgemeinerung gelten muß? 2. Es mag eine hagiographe Auseinandersetzung mit Cage geben allerdings $\mathrm{zu}$ behaupten, daß die Cage-Forschung auf eine solche unkritische Herangehensweise reduziert werden könnte, würde bedeuten, die bekannteste Veröffentlichung zu Cage nach 1992 - James Pritchetts The Music of John Cage, Cambridge, Cambridge University Press, 1993 zu ignorieren. Zwar mag sie nicht die kritischste Publikation zu Cage sein, enthält aber viele Kommentare, die weit von einer Hagiographie entfernt sind. Dazu zählt beispielsweise die Feststellung in der Einleitung des Buches, daß Cage in Apartment House 1776 (1976) die Zufallsprozeduren mehrmals ändern mußte, da er mit den daraus resultierenden klanglichen Konsequenzen nicht zufrieden war. Zwar stellt Pritchett nicht pointiert dar, daß dies einen Mythos bei Cage widerlegt. Dennoch kann seine Darstellung einer mehrmaligen Wiederholung von Zufallsprozessen folgende Fragen aufwerfen: Verhält sich so ein Komponist, der alle Klänge akzeptiert? Ist das ein Mensch, dessen Empfinden für klangliche Ereignisse inhuman abgestumpft ist? Diese Zuschreibungen sind ein offensichtlicher Cage-Mythos, der in dem von Mahnkopf herausgegebenen Buch nicht kritisch hinterfragt, sondern fast durchweg als Wahrheit argumentationslos bestätigt wird. 3. Cage hatte nicht eine Ästhetik und verfolgte nicht eine Philosophie, wie dies in MYTHos Cage oft stillschweigend angenommen wird. Wie lassen sich beispielsweise seine Interessen für Zen-Buddhismus, Erik Satie, Marshall McLuhan, Buckminster Fuller und Marcel Duchamp 
vereinbaren? Anstatt einer westlichem Denken entsprechenden Synthese, anstatt Dualismen, stehen bei Cage Pluralismen und unvereinbare Gegensätze - mitunter sogar Paradoxien — nebeneinander.

\section{$-3-$}

Barbara Zubers Beitrag ist der wissenschaftlichste und aufschlußreichste des Bandes. In The outcome is not foreseen - Le hasard sera notre dieu. Über John Cage und Antonin Artaud geht sie systematisch auf ein Zitat von 1966 ein, in dem Cage sein Werk in Verbindung zu Artauds Theaterideen bringt (Podiumsdiskussion der Tagung The Changing Audience for the Changing Arts/Panel, in: The Arts: Planning for Change, New York 1966). Mit höchster Akribie und argumentativ immer wieder belegt (was für diese Publikation einmalig ist), stellt Zuber zunächst Cages erste literarische Begegnung mit Artaud dar. Die Autorin verfolgt den ArtaudDiskurs zwischen Boulez und Cage und macht an ihm Unterschiede zwischen den beiden Komponisten fest. Sie kommt zu dem Schluß, daß Cage sich zwar auf Artaud berufe, seine musikalischen Konzepte aber nicht mit den Vorstellungen Artauds kongruieren. Daß Cage weder das symbolistische noch das mystische Moment Artauds in sein Schaffen integrieren wollte und daß daher sein Bezug zu Artaud keine vollständige Aneignung bedeuten kann, ist die Schlußfolgerung Zubers. In welcher Weise sich dieser Beitrag mit einem Cage-Mythos auseinandersetzt, wo doch die Musikwissenschaft, wie Zuber bemerkt, ,freilich, bis auf einige Streifzüge in dieses Gebiet, einen Bogen um Cages Artaud-Lektüre" (S. 223) gemacht hat, bleibt indessen unklar. Wie kann etwas ein Mythos sein, das bisher kaum von irgend jemandem beachtet wurde? Außerdem: Selbst wenn Cage sich permanent auf Artaud berufen hätte, wäre es kein Negativum, wenn sich seine Ästhetik nicht als Aneignung Artauds darstellen ließe. Mißverständnisse und Weiterentwicklungen von Theorien führen in Kunst und Musik zu Neuem; dort sind solche 'Denkfehler' gang und gäbe. So etwa in der Renaissance, in der durch die 'Aneignung' von Vorstellungen des Altertums und durch den Versuch, sich auf griechische Kultur zu beziehen, neue Musik und Kunst entstand.

\section{$-4-$}

Welche Mythen zu Cage kursieren und was diese uns über den Komponisten und seine Ästhetik sowie seine Musik sagen, wird auch in einigen der anderen Beiträge nicht immer deutlich. Beispielsweise geht Ian Peppers Artikel (John Cage und der Jargon des Nichts) intensiv auf Cages oft erwähnte Nähe zu ost-asiatischen Philosophien (besonders dem Zen Buddhismus) ein. Pepper intendiert, die Relation zwischen Cages Lehre bei Suzuki und der sogenannten Kyoto-Schule, zu der Suzuki gerechnet werden kann, aufzudecken. Ohne Zweifel ist Suzukis Einfluß auf Cages 
Denken wichtig. Auch macht es durchaus Sinn, wie Pepper zu fragen, wer eigentlich dieser Daisetz Taitaro Suzuki war. Dazu greift Pepper unter anderem auf eine Erörterung der Lehre Kitaro Nishidas zurück, da dieser als Begründer der Kyoto-Schule „Japans wichtigster Philosoph des zwanzigsten Jahrhunderts" (S. 17) und als Freund Suzukis sehr wichtig für die 'Lehr-Linie' Suzuki - Cage gewesen sei. Soweit wäre Peppers Gedankenkette noch nachvollziehbar; allerdings meint der Autor, auch Nishidas „wichtigsten Schüler“ (S. 20) hinzuziehen zu müssen: Keiji Nishitani. Daß dieser bei Heidegger zwischen 1936 und 1939 studierte, mag hier nur am Rande angedeutet sein, hat aber für Pepper eine entscheidende Interpretationskonsequenz: Nishitanis Philosophie „erinnert an Heidegger" (S. 21). Bei Nishitani "finden wir als Kern-Idee, daß echte 'Freiheit' nur aus blindem Gehorsam erwachsen könne; ergänzt mit der Empfehlung an uns [,...] auf unsere 'Befreiung' in resignativer Demut und mystischer Abgeschiedenheit in stiller Innerlichkeit zu erwarten [sic!]“ (S. 21). Wollte man Peppers Gedankensprung folgen, müßte Cages Ästhetik (die hier auf eine Aneignung der Ideen der Kyoto-Schule reduziert wird) als verwandt zu Nishitanis Philosophie verstanden werden. Dies trifft meiner Ansicht nach allerdings eher auf einen meditierenden Eremiten als auf Cages optimistisches, extrovertiertes Verhalten zu. Auch von seinen Hörern und Hörerinnen verlangte Cage nie 'resignative Demut und mystische Abgeschiedenheit' — eher das Gegenteil: die Zuhörer seiner Musik sollen im Hier und Jetzt des Alltages dessen visuelle und akustische Ereignisse als Kunst wahrnehmen. Aber Peppers Gedanken gehen weiter, ohne daß die Bezüge zu Cage deutlich werden: Das japanische Philosophentrio habe „,die Schlußfolgerung gezogen, daß nur eine feudalautoritäre Sozialstruktur dem Menschen die Möglichkeit bietet, als echtes Individuum zu leben" (S. 21). Von hier an präsentiert der Autor auf mehreren Seiten einen philosophischen Diskurs, der in sich schlüssig sein mag, dessen Relevanz für Cages Schaffen aber völlig unklar bleibt. Die Klammer (für Pepper) bildet Suzuki. Der Versuch einer Abgleichung von dessen diktatorischer japanischer Philosophie (so stellt sich Peppers Erörterung des Suzukischen Umfeldes dar) mit der Einschätzung, daß Cage ein autoritärer Denker und Komponist gewesen sei, ist nicht nachvollziehbar.

$$
-5-
$$

Daß Pepper seine Betrachtungen damit schließt, Cage quasi in eine gedankliche Ecke mit den Tätern von Auschwitz zu stellen, klingt so grotesk und entbehrt jedweder Logik, daß dieser Behauptung kaum argumentativ begegnet werden kann. Der Gedankengang Peppers, der zu einer solchen Schlußfolgerung führt, scheint (wie Zubers Beitrag) auf einer 
bestimmten Vorstellung von Einfluß und Aneignung $\mathrm{zu}$ beruhen. Das Prinzip ist folgendes: Ein Schüler (hier Cage) nimmt die Lehre seines Lehrers (hier Suzuki) uneingeschränkt auf. Weiter könnte ein Wissenschaftler nicht von Cages Ästhetik entfernt sein. Cages Philosophie ist ein widersprüchliches Konstrukt von Ideen und Ästhetiken, die sich auch bei folgenden in diesem Buch nicht wirklich erörterten Personen, die Cage beeinflußten, wiederfinden: Marcel Duchamp, Morton Feldman, Buckminster Fuller, Marshall McLuhan, Robert Rauschenberg, Erik Satie. Die Liste ließe sich fortsetzen. Wie die einfache Schnittmenge der Vorgehensund Denkweisen dieser Personen nicht Cages Ästhetik abbilden kann, so kann eine simplifizierte Herangehensweise an Cage nicht dem Menschen und Komponisten Cage gerecht werden.

Auch Frank Cox (Über John Cage) geht es um eine Vereinfachung der Einschätzung des Komponisten. Ausgehend von der Skizzierung des ,heiligen John Cage“ stellt Cox fest, daß dieser vor „, seiner Konversion und ,Wiedergeburt' [...] in der amerikanischen Musikwelt nicht besonders hoch angesehen "war (S. 36) Dieser Aussage fehlt eine genaue zeitliche sowie geographische Einordnung; ein auf den ersten Blick unwichtiger Kritikpunkt kann bei genauerer Betrachtung eine Menge an Problemen in Cox ${ }^{\text {* }}$ Darstellung aufzeigen, denn Cages Anerkennung als Komponist läßt sich nicht linear - zu einem Konversionspunkt hin — beschreiben. Nach anfänglichen Krisenjahren stößt erst Cages Verwendung des präparierten Klaviers auf eine positive Resonanz (er fungiert als erfinderischer, modernistischer Komponist, was in den USA Anklang findet). Als Cage danach, etwa ab 1951, mit Zufallsoperationen zu arbeiten beginnt, wandelt sich die Reaktion des Publikums - sein berühmtes 'stilles Werk' von 1952 kostet ihn die Freundschaft vieler Bekannter. Aufenthalte in Europa (in den 1950er Jahren) bringen ihm teilweise Anerkennung und lassen ihn andererseits sehr negative Reaktionen des deutschen Konzertpublikums erleben, bevor er den Kontakt zu 'Darmstadt' - bei seinem letzten Aufenthalt in den 1950er Jahren dort - bzw. zur deutschen Avantgarde abbricht. Erst 1958 mit dem sogenannten 25 years-retrospective concert will sich in den USA wieder eine größere Zahl an Menschen mit seinem Werk auseinandersetzen. Ab 1958 nimmt Cage vor allem seine Chance wahr, durch Publikationen (besonders durch seine gesammelten Schriften) und Vorträge seine Philosophien stärker zu verbreiten. Er setzt seine Bekanntheit ein, um anderen Künstlern zu helfen (beispielsweise der Merce Cunningham Dance Company) und nutzt viele Stunden seiner freien Zeit nicht zum Komponieren, sondern auch dazu, durch Korrespondenzen mit Mäzenen Geld für andere künstlerische Projekte zu sammeln (etwa durch 
das Notations-Buch; hrsg. mit Alison Knowles, Something Else Press, New York, 1969). Er nimmt Lehrverpflichtungen wahr (die oft eher eine Residenz an einer Universität ohne festgeschriebene Aufgaben und Dozentenpflichten bedeuten) und widmet sich auch verstärkt dem Schreiben von Mesostics (diese sind poetische Texte, deren formaler Aufbau durch ein vertikal durch die Mitte jeder Zeile verlaufendes Wort geprägt sind) und anderen Textgattungen. Von etwa 1982 bis zu seinem Tode im Jahre 1992 kann sich Cage vor Kompositionsaufträgen und Anfragen nach Interviews, Vorträgen und schriftlichen Äußerungen kaum retten. Obwohl nach seinem Tod in gewissen Zirkeln eine Art 'Heiligenverehrung' stattfindet, kann von einer breiten Vereinnahmung Cages für eigene kompositorische Zwecke (etwa durch jüngere Komponisten, die ihr eigenen Experimente durch Cages Schaffen legitimiert sehen) oder zur Propagierung der Neuen Musik keine Rede sein. Dennoch formuliert Cox folgende Kritik an Cages Werk und Denken (S. 50-51):

1. Ästhetisch: Cage habe eine „Gleichgültigkeit gegenüber den Imperativen einer bestimmten Kunstform " an den Tag gelegt (S. 50). Hier geht es Cox primär darum, daß Cage alle Zufallsergebnisse akzeptiert habe (daß dies nicht der Fall war, habe ich bereits erwähnt).

2. ,Philosophisch/gesellschaftstheoretisch': Cox glaubt, daß Cage durch sein Beharren auf Grundsätzen faktisch eine Religion mit Glaubensgrundsätzen geschaffen habe (,die oft zu faschistischen Tendenzen führten"). Dies mag so wirken. Auch wenn Cages Denkweisen als ein relativ hermetisches Denksystem verstanden werden können, so wäre es doch möglich, Ähnliches bei vielen anderen Komponisten $\mathrm{zu}$ finden.

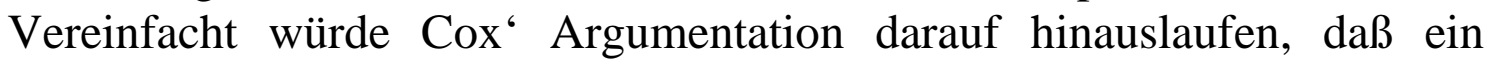
Mensch mit starken Überzeugungen faschistische Tendenzen in seinem Denken hat. Daß Cage selbst nicht immer so dogmatisch handelte, wie er 'predigte', und sich auch oft widersprach bzw. im Laufe seines Lebens seine Einstellung änderte, zeugt davon, daß viele seiner Äußerungen zwar unflexibel wirken mochten, er selbst es aber nicht bei allen seiner Ansichten war.

3. Kritik an der 'Neuen Musik-Szene', die Cage für ihre Zwecke benutzt habe: Wie Cox selbst andeutet, gilt dieser Kritikpunkt „weniger Cage selbst als vielmehr seinem Einflu $\beta$ gerade in Amerika, [wo seine Philosophie herangezogen wurde; C.G.], um eine ganze Reihe halbfertiger ,Experimente" zu rechtfertigen" (S. 51). Cage hat mehrmals darauf hingewiesen, daß er nie für alle Experimente anderer Komponisten die 
,Erlaubnis' gegeben habe (beispielsweise in einem Interview mit Peter Gena: John Cage/Peter Gena, After Antiquity. John Cage in Conversation with Peter Gena, in: A John Cage Reader in Celebration of his 70th Birthday, hrsg. von Jonathan Brent, Peter Gena und Don Gillespie, New York, C. F. Peters Corporation, 1982, S. 167-183; hier S. 171-172).

\section{-7 -}

Berthold Tuerckes Aufsatz Einfallswinkel wider Zufallswinkel. Über Tauschwege und Täuschungsweisen in der Alchimie von John Cage erweckt fast den Eindruck, als ziele der Autor darauf ab, Cage als einen unseriösen Gaukler zu beschreiben. Oberflächlich betrachtet, erörtert der Autor Ideen einzelner Werke und mögliche Interpretationsansätze, etwa anhand der Beschreibung einer Probe der Thirty Pieces for String Quartet durch das Kronos Quartett. Ein Beispiel für den streckenweise bizarren Stil des Autors soll hier aber nicht vorenthalten werden (das Layout folgt dem Original):

„Schönbergs alter Traum, den er einst Busoni offenbart hatte, wird wahr: 'völlige Freiheit von allen Formen'.

Schönberg, ein Anarchist?

Ein halber, gewiß.

Und halb wahr wird auch der Traum bei Cage. “ (S. 62)

Andere Aussagen werden wahrscheinlich ebenso ewig auf eine nachvollziehbare Beweisführung Tuerckes warten müssen: „So tickt das Gros der Cageschen Musik stets unterm selben Tempo 60 des Sekundenzeigers. " (S. 63). Sich über solche Zeilen — in ihrer anmaßenden Generalisierung ebenso unzutreffend wie eine mögliche Behauptung, daß Schönberg 'immer atonal' komponiert habe - zu wundern, ist nicht erstaunlich. Erstaunlich hingegen ist, was hinter all den aphoristischen Andeutungen Tuerckes durchzuschimmern scheint: 1. Daß Cages Zufallsoperationen selbst nicht immer eine intendierte, mit Vorlieben behaftete Klangvorstellung verhindern (vgl. S. 67 sowie S. 73-74) und vielmehr eine Scheu vor der kompositorischen Auseinandersetzung bzw. vor der Bejahung des Subjekts offenbaren (vgl. S. 72-73). 2. Daß daraus Konsequenzen für eine Interpretation zu ziehen seien (vgl. S. 73-74). Leider werden diese aufschlußreichen Aspekte der Cageschen Kompositionsweise und der Aufführungspraxis seiner Musik von Tuercke nicht so dargestellt, daß die Auseinandersetzung mit dem Mythos des alles negierenden Cage evident wird. Tuerckes unklare Sichtweise auf Cage und dessen Schaffen zielt vielmehr auf Zeilen wie die folgende: „Letzerem [einer Anarchie ins Chaos; C.G.] hat Cage selbst tüchtig Vorschub geleistet durch das anything goes in 
vielen der Spielanweisungen, die er seinen Stücken voranstellt." (S. 62) Diese Vorstellung Tuerckes ist selbst ein Mythos - Cage unterschied sehr wohl zwischen guten und schlechten Interpretationen seiner Werke (ein Beispiel hierfür bildet etwa eine Aufführung der Europeras 1+2 durch das Zürcher Opernhausorchester; siehe Cages Brief an dieses Ensemble in Richard Kostelanetz (Hrsg.), John Cage, Writer, New York, Limelight Editions, 1993, S. 255-256). Ein Interview aus dem Jahr 1976 belegt außerdem, daß Cage ein „Alles geht schon" nicht wollte und sich auch dementsprechend äußerte (Tracy Caras/Cole Gagne, An Interview with John Cage (1975), in: New York Arts Journal, Jg. 1, 1/1976, S. 7).

$-8-$

Johannes Bauers Ausführungen stehen unter der Überschrift Cage und die Tradition. Leider spiegelt sich die Klarheit des Titels nicht in Bauers Text wider: Cages Bezug zu nur einer einzigen Tradition ist unmöglich herstellbar; da dieses Buch aber von den Mythen von, um und über Cage handelt, sollte doch zwei vom Komponisten selbst propagierten Bezügen seines Schaffens und Denkens nachgegangen werden. Cages mögliche Bezüge zur europäischen Tradition (sei es zur Denkweise seiner kurzzeitigen Lehrer Schönberg und Weiss) oder auch zu früheren 'Vertretern' der sogenannten American Experimental Music (in erster Linie Charles Ives und Henry Cowell) wären naheliegende Ansatzpunkte einer Auseinandersetzung mit zwei von vielen Traditionen, zu denen Cage in bestimmten Abschnitten seines Lebens direkten Kontakt hatte. Statt dessen erwähnt Bauer Perotin, Josquin, Adam von Fulda und Beethoven. Anstatt naheliegende Bezüge des Cageschen Schaffens zu erörtern (zu Cowell und Ives; immerhin wird letzterer auf S. 97 kurz erwähnt), verheddert Bauer sich in teilweise ausschweifenden Vergleichen mit Karlheinz Stockhausen, Pierre Boulez und Iannis Xenakis. Nicht, daß solche Querverbindungen nicht aufschlußreich sein könnten, doch auch wenn Bauers Vergleich der Cageschen Philosophie mit derjenigen Nietzsches, Hegels, Schlegels oder Baudelaires viele diskussionswürdige Punkte einer Auseinandersetzung anschneidet, verliert sich der Fokus in den 52seitigen Ausführungen. Durch weniger umfangreiches und stärker fokussiertes Arbeiten hätte Bauer gewiß eine überzeugendere Darstellung erreicht.

\section{$-9-$}

Zufall und Subjekt. Erwägungen zu Cage von Larson Powell ist ein gutes Beispiel für einen gravierenden Fehler der vorliegenden Sammlung von Beiträgen. Powells Text liest sich wie eine Wiederholung bereits erwähnter Aufsätze dieser Publikation: Es geht nochmals um den Zufall und das Subjekt, ein Aspekt, der auch bei Zuber eine große Rolle spielt. Erneut, wie bei Bauer und Zuber, werden viele Vergleiche mit Boulez gezogen. Eine 
Besonderheit Powells ist jedoch, daß er auf dem Höhepunkt seiner Argumentationslinie über Cage behauptet, er habe die 'totale Negation' in seinen Kunstwerken angestrebt (S. 213). Für Powell scheint dies durch eine Abkehr vom Subjekt - d.h. dadurch, daß Cage keine kompositorischen Entscheidungen getroffen und der Zufall den 'künstlerischen Schaffensprozess' übernommen habe - ausreichend belegt zu sein. Der Autor muß sich fragen lassen, wie etwas, das als Komposition unter dem Namen eines Menschen als Kunstwerk aufgeführt und verkauft wird, eine 'totale Negation' sein kann. Außerdem sind viele Kompositionen Cages alles andere als eine Negation (dazu mehr weiter unten). Auch wenn Powells Behauptung nicht gerade philosophisch durchdacht ist, ließe es sich mit solch einer Aussage leben - allerdings setzt Powell zu einem wissenschaftlichen Faux-pas an, der kritisiert werden muß. Von seiner nicht überzeugenden Feststellung hangelt er sich zu einem Modell von Jacques Lacan (Analyse des Fetischismus), welches besagt, daß anstelle eines Werkes, in dem sich ein Subjekt ausdrückt, am Ende ein Nichts übrigbleibt (d.h. am Ende des Schaffensprozesses des Komponisten steht keine Komposition, sondern 'Nichts'). Für Powell belegt diese Applikation des Lacanschen Modells, daß Cage tatsächlich die "totale Negation“ anstrebte (vgl. S. 213-214). Dies ändert aber nichts an der Tatsache, daß die ursprüngliche Behauptung des Autors nicht in sich belegt ist. Will sagen: Es wird nicht klar, warum er Cages Schaffen auf diese Weise interpretiert. Dabei scheint Powell sich auf einen stillschweigenden Konsens der Autoren der gesamten Publikation zu beziehen: Cage habe eine totale Negation angestrebt (angeblich mustergültig erkennbar in den berühmten 4'33") und daher am Ende des Schaffensprozesses Nichts produziert. Daß diese Denkweise selbst unkritisch einem Mythos der anti-cageschen Wissenschaftsgemeinde folgt, sich aber leicht - in ihrer Totalität widerlegen läßt, kann mit einem Hinweis auf Werke wie Cheap Imitation (1969) oder auch die ab 1987 komponierten number pieces klar gezeigt werden. Während Cheap Imitation lediglich die Folgerichtigkeit der Tonalität negiert, aber die Phrasenstruktur von Saties Sokrates beibehält, sind viele der number pieces akustisch kaum von anderer moderner Musik pointillistischer Prägung unterscheidbar (in ihrer Art der Aufführung negieren sie partiell die Allmacht des Komponisten und eliminieren die Notwendigkeit eines Dirigenten).

$$
-10-
$$

$\mathrm{Da}$ weder die Cage-Forschung noch das homogene Euvre bei Cage existiert, wird in MYTHos Cage fast durchweg von den Autoren und der Autorin übersehen. Einzig Richard Toop hat klar erkannt, daß es , gleichsam mehrere John Cages gab, und daß wir immer, wenn sich jemand 
kommentierend auf John Cage bezieht, fragen dürfen, welcher John Cage bzw. der John Cage welcher Zeit gemeint sei“ (S. 181). Toops John Cage gegen seine Aneigner verteidigt ist eine knappe Analyse des Versuches einer ästhetisch-ideologischen Einordnung Cages sowie von dessen Vereinnahmung durch einige Wissenschaftler. Toop geht der Frage nach, ob Cage nun ein Vertreter der Postmoderne oder der Moderne gewesen sei. Dabei setzt er sich mit verschiedenen Mythen der Cage-Rezeption auseinander (die von Toop erwähnten Publikationen zeichnen sich durch Ungenauigkeiten oder Generalisierungen aus), was zu manchen erkenntnisreichen Gedankengängen führt.

$-11-$

Toops Beitrag ist eine leicht überarbeitete Übersetzung eines englischen Artikels (John Cage Defended against his Appropriators, in: Literature and Aesthetics, Jg. 3, 1993, S. 96-107). Dies wäre an sich nicht schlimm, wenn die Übersetzung fehlerlos wäre. Daß die Übersetzerin nicht aus der deutschen Ausgabe von Daniel Charles' For the Birds zitiert (London, Marion Boyars, 1981), sondern die englischen Zitate übersetzt, mag nur die deutschen Leser verärgern, die nach den angegebenen Seitenzahlen der englischen Ausgabe versuchen, Zitate in der deutschen Fassung zu finden (dies gilt ebenso für Kostelanetz' Conversing with Cage und Silence, die in deutschen Editionen vorliegen). Daß aber ,indeterminate work“ (Daniel Charles/John Cage, For The Birds, S. 79) mit 'nicht determinierte Musik' übersetzt wird, ist einer der Übersetzungsfehler dieses Textes. Der Unterschied zwischen 'nicht determiniert' und 'unbestimmt' mag marginal anmuten, ist jedoch entscheidend. Während viele Werke Cages nach 1950 indeterminate komponiert wurden (zum Beispiel durch Zufallsoperationen), sind doch einige Notationen dieser Kompositionen sehr exakt und determiniert (etwa die Freeman Etudes, 1977-1980/1989-1990). Während die Notation und der Kompositionsprozeß einiger Stücke sehr determiniert ist (das 'stille Werk' von 1952 oder O'00" von 1962 wären zwei gute Beispiele), ist die Ausführung (d.h. der exakte Klang) unvorhersehbar, also unbestimmt. Wer traute einer primär deutschlesenden Zielgruppe nicht die Lektüre eines oder mehrerer Beiträge auf Englisch zu?

$$
-12-
$$

Till A. Körber setzt in seinen Gedanken über das Hören anläßlich von Cageerlebnissen die eigene Hörerfahrung als Ausgangspunkt für eine dilettantische Analyse des eigenen Hörens Cagescher Musik. Da er in seinen neunseitigen Betrachtungen nie ein einziges Werk von Cage erwähnt, sollten seine generalisierenden ‘Gedanken' unter die Kuriositäten der Cage-Literatur eingestuft werden. 
Günter Seubold betitelte seinen Aufsatz mit einer Aneinanderreihung von Worten, die von vornherein die Seriosität seines Vorhabens in Frage stellen: Verdinglichter Zufall, Verräumlichte Zeit, Weiße Stille. John Cage oder Wie man dem Gefängnis westlicher Musik durch östliche Anleihen entgehen will und dabei doch nur den endgültigen Sieg der abendländischen Bild- und Präsenz-Ästhetik über die sich zu befreien suchende Musik erringt. Wie der Titel einen unklaren und pompösen Eindruck hinterläßt, so ist leider auch der übrige Beitrag nicht klar und faßbar. Deshalb bleibt nur noch das Schlußwort Seubolds hinzuzufügen: „Doch aufgrund der soeben angeführten Aspekte scheinen Adornos Musikästhetik und die Komponisten, die sich seinem Autonomie-Ansatz auch heute noch verpflichtet fühlen, weitaus fortschrittlicher zu sein. Ihnen dürfte eher die Zukunft gehören als Cages - temporär interessanten [sic!] — Versuchen." (S. 177) Solch philosophisch angereicherte Feuilletonkritik an Cage deckt keine Mythen auf, sondern macht sich im wissenschaftlichen Kontext lächerlich.

Auch der Beitrag Claus-Steffen Mahnkopfs zielt vorwiegend auf eine Einschätzung des Nachwirkens von John Cage ab. Während dieses Vorhaben, obwohl sehr konventionellen und traditionellen Denkmustern folgend, per se nicht ohne Erkenntnisgewinn bleiben muß, überzeugt Cages kompositorische Hinterlassenschaft besonders wenig in zwei Punkten. Erstens: Unter der Kapitelüberschrift 'Zeugnisse' will Mahnkopf anhand einiger ausgewählter Autoren und deren Publikationen aufzeigen, wie diese — in Mahnkopfs Augen - fehlerhaften „Projektionen, die auf Cage, eine leere Plattform ohne Geometrie, gerichtet werden “ (S. 133), zu widerlegen sind. Mahnkopf geht davon aus, daß die von ihm ausgewählten Autoren etwas auf Cage projizieren, was er eigentlich nicht realisiert habe: die Befreiung von Hierarchien und Machtverhältnissen. Ich möchte nur kurz auf zwei der von Mahnkopf besprochenen Autoren eingehen: Er bezieht sich etwa auf Daniel Charles' Artikel La paume (de) la dent (in: John Cage, München, text + musik edition, 1990) und stellt fest, daß Charles „mit dem entscheidenden Kategorienfehler aller Cageianer, der Behauptung einer Hierarchie, die keine ist", beginnt (S. 138). Während seine Kritik an Charles etwas nebulös bleibt (sie zielt eigentlich vorwiegend darauf ab, daß Charles keine „Grundkenntnisse“ von Musik besitze; S. 139), ist sie bei Heinz-Klaus Metzger anmaßend direkt. Mahnkopf zitiert zunächst Metzgers Position (Heinz-Klaus Metzger, John Cage oder Die freigelassene Musik, in: John Cage I, München, text + musik edition, 1990, S. 5-17) wie folgt: 
„Erst die Musik von Cage durchbräche die mythische Konitinuität der Naturbeherrschung, ja von Beherrschung insgesamt. [...] Erst Cage führe zu Ende, was Telos seit der Emanzipation der Dissonanz war: die Befreiung jedes einzelnen Klangereignisses, ja die Befreiung von Ordnung, und dies heißt vor allem: Befreiung von der Ordnung der Zeit-Sukzession [...], d.h. der (in diesem Falle zufallsgesteuerten) Verteilung des Materials auf den Zeitverlauf befreie das Einzelne von der Hierarchie der Abfolge. " (S. 135)

Mahnkopfs Kritik folgt prompt:

„Der Gedanke ist so einfach wie falsch. Jedes Komponieren, und sei es einer einzelnen Note, sei bereits Ausdruck von Macht und somit von Unfreiheit. Das mag überzeugend nur auf den wirken, dessen psychische Not ihn zur Sucht allzu einfacher Lösungen treibt. Denn Okkurenz ist genausowenig ein Parameter musikalischer Produktion wie das Sein ein Prädikat Gottes. [...] Metzgers Grundfehler liegt auf der Hand: Im Namen von ,Herrschaftsfreiheit'verdammt er alle Ordnung, und eben deswegen entgehen ihm gerade jene realen Zwangssituationen, die beim Namen genannt werden müßten. Auschwitz war eine Zwangssituation, nicht die phonetische und morphologische Ordnung im Wort, Ordnung '. [...] Warum ist Musik, die als Schrift notiert ist, per se Ausdruck von Gewalt, Herrschaft und Verderben? Eine solche These ist gerade für den, der philosophisch zu denken vermag, derart absurd, daß man fragen mu $\beta$, worum es eigentlich geht, wenn es beim Cageianismus nicht um Musik geht. Denn, daß etwa die Musiker nun plötzlich machen dürften, was sie wollten [...], als die höchste Form der Musikentwicklung anzubieten, ist nicht nur hirnrissig, sondern gerade nicht Ausdruck von Freiheit, sondern regelrecht von Unfreiheit, Ausdruck der Unterdrückung dessen, was Musik so unbeschreiblich göttlich macht. "(S. 135-136)

Diese Beobachtung ist aus mehreren Gründen mehr als fragwürdig: 1. Mahnkopf widerlegt nirgendwo in seinem Artikel argumentativ, daß Cage Hierarchien aufgebrochen habe - er behauptet einfach das Gegenteil von dem, was die 'Cageianer' 'behaupten' (Gegenbeispiel soll hier das Concert for Piano and Orchestra sein. Es ist eindeutig eine Abkehr von 
Hierarchien: Der Dirigent kontrolliert nicht den musikalischen Fluß, sondern fungiert lediglich als Chronometer; solch eine sachliche Auseinandersetzung mit der Musik selbst — das heißt mit den Kompositionen Cages - präsentiert Mahnkopf nicht.). 2. Der eigentliche Mythos, den Mahnkopf übersieht, ist das Paradoxon bzw. die Utopie der Situation jeder Kunstausführung: Wie jede Aktion eines Menschen, jede Intention sich auf Machtverhältnisse, strukturelle Gewalten und unfreie Konstellationen (Hierarchien) beziehen läßt, so unterliegt auch jede Kunstausübung diesen Gegebenheiten. Ebenso wie Beethovens, Schuberts und Wagners Kompositionen besitzen auch Cages Werke Hierarchien (besonders deutlich wird dies bei Werken für größere Ensembles: Die Musiker und Musikerinnen stehen unter der Leitung eines Dirigenten, der die Intention des Komponisten durchsetzt; eigene Entscheidungen der Interpretation der Notation sind nicht gefragt). 3. Allerdings gelang es Cage in einigen seiner Kompositionen, die traditionell sehr fixierte Konstellation zwischen Komponist, Interpret und Zuhörer aufzulösen (4'33“ aus dem Jahre 1952 ist eben nicht einfach nur Nichts, sondern genau der Versuch einer Auflösung der Funktion des Komponisten und ansatzweise des Interpreten; die späten, sogenannten number pieces (1987-1992) bieten dem/den Interpreten die Möglichkeit, eine 'kontrolliert' anarchische musikalische Situation zu erzeugen, ohne daß ein Dirigent oder Cage als Komponist Einfluß auf die exakte Klangstruktur der Aufführung haben kann.). 4. Hierarchien in moderner Kunstmusik des 20. Jahrhunderts existieren auch durch oft bis ins letzte Detail auskomponierte, komplexe musikalischstrukturelle Systeme (Komplexismus; bei Cage existieren solche Klangwelten teilweise auch ganz ohne determinierte Formungen des Komponisten: etwa in Musicircus (1969) als Additionen von einzelnen, nicht durch einen 'Metaplan' koordinierten musikalischen Ereignissen; ob dieser kompositorische Unterschied hörbar ist, darf bei einer erhöhten Menge an 'Klanggeweben' bezweifelt werden). Der Unwissende ist ausgegrenzt, der 'ungelernte' Zuhörer muß erst die Partitur oder das Repertoire studieren, bevor er/sie das Werk verstehen kann. Viele von Cages Kompositionen behaupten nicht, mehr zu sein, als sie sind: $\mathrm{Zu}-$ sammenklänge ohne eine symbolische oder systematisch-strukturale Interpretationsschicht. Von einer Musik aber genau dies zu verlangen (wie es scheinbar als Dogma und Entscheidungshilfe für 'gute Musik' bei Mahnkopf oberste Prämisse ist), bedeutet, Musik und Kunst auf die Vermittlung von emotionalen und/oder rationalen Aspekten zu reduzieren. Fast alle musikalischen Werke Cages haben keine zweite semantische Schicht, keine musikalische Geheimsprache, die erst noch dechiffriert werden muß. Alleine durch ihren kompositorisch-philosophischen Ansatz sind daher, 
theoretisch gesehen, viele Cageschen Kompositionen auditiv leichter für den Hörer erfaßbar als andere Musik des 20. Jahrhunderts, da in ihnen keine musik-immanenten Aussagen existieren. 5. Musik als „göttlich“ zu bezeichnen, mag Komponisten der Romantik, die an Konzepte wie Inspiration glauben, zugestanden werden - von einem wissenschaftlich analysierenden Komponisten des 21. Jahrhunderts geäußert, befremdet eine solche Aussage.

\section{$-15-$}

Mahnkopf gibt zu, daß die Auswahl der von ihm analysierten Publikationen zu Cage „zwar selektiven Charakters [...], aber durchaus repräsentativ" sei (S. 133). Es ist dabei nicht klar, warum sich Mahnkopf nur mit jeweils einer Publikation von Hans Heinrich Eggebrecht, Reinhard Oehlschlägel, Daniel Charles, Stefan Schädler und Jean-François Lyotard auseinandersetzt. Wie bereits für Charles angedeutet: Warum wird das Denken eines Autors auf eine Publikation reduziert? Außerdem: Warum wird zum Beispiel kein Bezug genommen auf James Pritchetts The Music of John Cage, Cambridge, Cambridge University Press, 1993, auf David Wayne Pattersons Nachruf (Cage and the New Era, in: Repercussions, Jg. 1, 2/1993, S. 5-30), auf Pattersons Dissertation (Appraising the Catchwords, c. 1942-1959: John Cage's Asian-Derived Rhetoric and the Historical Reference of Black Mountain College; Columbia University, Ph.D. diss., 1996) oder auf Moira Roths Five Stories about St. John. Seven Stories about St. Pauline. Surely There is Trouble in the John Cage Studies Paradise. And Readings From Today's Headlines of the New York Times (in: Moira Roth: Difference/indifference: Musings on Postmodernism, Marcel Duchamp and John Cage, Amsterdam, G and B Arts International, 1998, S. 137 - 143)?

$$
-16-
$$

Der zweite Punkt meiner Kritik geht an die eigentliche Substanz - die Auseinandersetzung mit Cages Kompositionen, d.h. auch den Partituren, selbst - und läßt die Vermutung zu, daß Mahnkopf wenig Sorgfalt und Akribie in diesen Beitrag investiert hat. Zwei Sätze sollen als Beleg meiner Kritik genügen: „Daß 4'33“ eine Schlüsselkomposition des 20. Jahrhunderts sei, spricht viel [sic] dieses Jahrhundert, ähnlich Stalin und der Atombombe, ist geradezu das Symbol für das Scheitern der Neuen Musik, deren bekannteste Kultfigur Cage immer noch für den Großteil derer ist, die Neue Musik nicht kennen. " (S. 147) Abgesehen davon, daß dieser Satz grammatikalisch keinen Sinn macht, muß doch sehr bezweifelt werden, daß Cage eine Kultfigur für Nichtkenner der Neue Musik-Szene ist. Was Stalin, die Atombombe und Cage gemeinsam haben, erklärt Mahnkopf nicht. Der zweite Satz zeugt schlicht von unglaublicher Unwissenheit: „Cages 
'Ästhetik' fokussiert sich im Nichts, die 'Komposition' 0'00" ('Solo, das von irgend jemandem auf irgendeine Weise aufzuführen ist', übrigens identisch mit jedem je komponierten Solowerk), ist Cages musikalisches Vermächtnis [...] “ (S. 147). Daß Cages 0'00“ noch sieben weitere Anweisungen enthält, die Rahmenbedingungen für dieses Solo setzen, muß Mahnkopf entgangen sein, sonst würde er seine Behauptung nicht aufstellen können.

$-17-$

Abschließend: Die Aufsatzsammlung MYTHos Cage verheddert sich in der Auseinandersetzung mit ihrem Gegenstand. Anstatt Cage-Mythen auf wissenschaftliche Weise darzulegen und $\mathrm{zu}$ dekonstruieren, werden bestehende Klischees weitestgehend wiederholt. So zielt das Buch darauf ab, glorifizierende bzw. positive Ideen der 'Cageinaer' anzuprangern, ohne sie (beispielsweise die fragwürdige 'hagiographe' Darstellung Cages) argumentativ zu widerlegen. Statt dessen werden lediglich 'negative Mythen' von Cage-Gegnern - etwa Cage als einen ikonoklastischen Komponisten aufzufassen, der in seinem Euvre alles negiert — der vermeintlichen 'Cage-Hagiographie' entgegengestellt. Für den Leser, der noch nie etwas über/von Cage gehört oder gelesen hat, ist dieses Buch aufgrund der in den meisten Beiträgen schlecht informierten, unwissenschaftlichen Vorgehensweise nicht empfehlenswert. Auch der Leser oder die Leserin, der/die über Vorkenntnisse verfügt, kann von diesem Buch kaum profitieren, da die Lektüre einer Vielzahl von Seiten wenig erkenntnisreich ist. 